\title{
Acute Stroke Treatment in Patients with Basilar Artery Occlusion: A Single-Center Observational Study
}

\author{
Isabella Francalanza ${ }^{a}$ Antonio Ciacciarellia Antonio Armando Caragliano ${ }^{b}$ \\ Carmela Casella $^{a}$ Masina Cotroneo $^{a}$ Cristina Dell'Aera $^{a}$ \\ Maria Carolina Fazio ${ }^{a}$ Francesco Grillo $^{a}$ Antonio Pitrone ${ }^{b}$ \\ Sergio Lucio Vincib Giuseppe Trimarchic ${ }^{\mathrm{c}}$ Rosa Fortunata Musolino ${ }^{a}$ \\ Paolino La Spina ${ }^{a}$ \\ aStroke Unit, Department of Clinical and Experimental Medicine, University of Messina, \\ Messina, Italy; ${ }^{b}$ Neuroradiology Unit, Biomedical Sciences and of Morphologic and

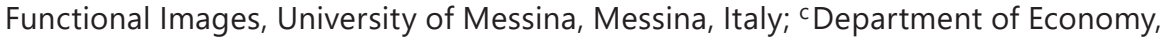 \\ University of Messina, Messina, Italy
}

Keywords

Acute ischemic stroke - Basilar artery occlusion - Posterior circulation ASPECTS · Collateral circulation

\section{Abstract}

Background: Acute ischemic stroke (AIS) due to basilar artery occlusion (BAO) represents $1-4 \%$ of all ischemic strokes. BAO results in strokes associated with a high risk of a poor functional outcome and, in $86-95 \%$ of the untreated cases, it results in death because of the vital cerebral structures involved. Diagnosis can be delayed because of the variability in presenting symptoms, and acute treatment is often attempted even beyond $6 \mathrm{~h}$ from symptoms onset because of the high risk of a fatal prognosis. Objective: In this observational study, we retrospectively analyzed patients with AIS due to BAO referred to the stroke center of the University Hospital of Messina. We aimed to assess prognostic factors and to evaluate the association between clinical outcome and posterior circulation Alberta Stroke Program Early CT Score (pc-ASPECTS) and collateral status. Method: BAO was confirmed by cerebral computed tomography (CT) angiography or cerebral angiography. All patients underwent CT scan and magnetic resonance imaging (MRI). We assessed the pc-ASPECTS on diffusion-weighted imaging (DWI) MR images and the Posterior Circulation Collateral Score (PC-CS) for every patient. Functional outcome was assessed at 3 months using the modified Rankin Scale (mRS). Results: The study population consisted of 27 patients; 16 males and 11 females. The mean age was 66 ( \pm 14 ) years. We observed a favorable outcome (mRS $0-3)$ in $40.7 \%$ of cases, $25.9 \%$ reached mRS $0-2$, and $29.6 \%$ had a poor clinical outcome (mRS 4-5). Patient survival was 
$70.4 \%$, whereas 8 patients died (29.6\%). In 7 patients, pc-ASPECTS was $\geq 7$. According to the PC-CS, 33.3\% had moderate collaterals and $63.0 \%$ had good collateral status prior to receiving the treatment. Favorable outcome was significantly associated with age, NIHSS score at admission, pc-ASPECTS, hypercholesterolemia, and female sex but not with the other risk factors. Conclusions: In our study, we found that younger age, low NIHSS score at admission, and high pc-ASPECTS, but not onset to treatment time, are associated with a favorable clinical outcome. Transferred patients did not have a significantly poorer outcome. These findings confirm that acute stroke treatment improves clinical outcome in BAO patients, in spite of a delayed diagnosis and an extended therapeutic window, considering lesion volume and localization in DWI MRI.

\section{Introduction}

Acute ischemic stroke (AIS) due to basilar artery occlusion (BAO) represents $20 \%$ of posterior circulation strokes [1] and 1-4\% of all ischemic strokes [2]. Acute BAO is associated with severe neurological deficit and is potentially fatal. The mortality rate, if BAO is not treated, is up to $86 \%$ [3-6], and the rate of good clinical outcome without recanalization is approximately $13 \%[1,7]$. The spontaneous recanalization rate is thought to be low and not to exceed $20 \%$ [8]. BAO diagnosis can prove difficult because of the variability in clinical presentations, and stroke onset can be abrupt or preceded by dizziness, vertigo, and nausea $[2,9,10]$.

There is no consensus on the best therapeutic approach for acute BAO [11-13]. For instance, a recent meta-analysis observed a superiority of endovascular treatment [14]. Because of the high risk of a poor outcome and a fatal prognosis, acute treatment is often attempted beyond $6 \mathrm{~h}$ [3], sometimes reaching 12-24 h from symptoms onset [5].

In this observational study, we retrospectively analyzed patients with AIS due to BAO referred to the stroke center of the University Hospital of Messina that serves an area encompassing the whole of Sicily. Few studies have examined predictors of functional outcome in patients with $\mathrm{BAO}$, and poor data are available regarding the management of these patients. We report a single-center retrospective case series of $\mathrm{BAO}$ patients. We aim to evaluate prognostic factors and the association between clinical outcome and posterior circulation Alberta Stroke Program Early CT Score (pc-ASPECTS) [15] and collateral status.

\section{Materials and Methods}

A retrospective analysis was performed on patients with BAO confirmed by cerebral computed tomography (CT) angiography or cerebral angiography, referred to our stroke center from 2014 to 2018, who underwent acute stroke treatment. Acute treatment decision was made case-by-case by the vascular neurologist and the neuro-interventional radiologist, considering clinical and imaging data. Mainly, patients were considered not eligible in case of profound coma, bilateral mydriasis, and absence of brainstem reflexes. Patients were excluded if clinical follow-up information was not available.

Therapeutic approaches included thrombo-aspiration, intra-arterial thrombolysis, thrombectomy, or a combination of these, based on the evaluation of the single case. Intravenous thrombolysis (IV-T) with recombinant tissue plasminogen activator was administered to eligible patients. 
Collected data included patientcharacteristics, symptoms onset, stroke severity as expressed by the National Institutes of Health Stroke Scale (NIHSS) score on admission and discharge, and the modified Rankin Scale (mRS) at 3 months. Onset to treatment time (OTT) was defined as the time between symptom onset and the beginning of IV-T or femoral artery puncture.

All patients underwent a diagnostic brain CT scan and brain magnetic resonance imaging (MRI). We assessed the pc-ASPECTS on diffusion-weighted imaging (DWI) MRI for every patient [15]. pc-ASPECTS is a 10-point scoring system to asses early ischemic changes in the posterior circulation [16]. Collateral status was evaluated using the Posterior Circulation Collateral Score (PC-CS) on angiographic images [17]. The PC-CS is a 10-point grading system that evaluates the collateral flow in the posterior circulation in patients with acute BAO. It takes into account the patency of the posterior inferior cerebellar artery, anterior inferior cerebellar artery, and superior cerebellar artery, assigning 1 point for each of them. One point is allocated if the posterior communicating artery diameter is smaller than the ipsilateral first segment (P1) of the posterior cerebral artery and 2 points are allocated if the diameter is equal or larger than the ipsilateral P1 segment diameter. Collaterals were categorized as poor (PC-CS 0-3), intermediate (PC-CS 4-5), or good (PC-CS 6-10) [17]. Recanalization status was classified according to the Thrombolysis in Cerebral Infarction (TICI) classification at the end of the procedure, and successful recanalization was defined as TICI score $2 b-3$ [18].

CT imaging was routinely performed at approximately $24 \mathrm{~h}$ after treatment, and intracranial hemorrhage was classified as hemorrhagic infarction on follow-up imaging. Stroke etiology was defined according to the Trial of Org 10172 in Acute Stroke Treatment (TOAST) criteria [19].

We assessed functional outcome at 3 months using the mRS during a follow-up outpatient visit or via a semi-standardized telephone interview. Favorable outcome was defined as mRS 0-3, in accordance with the definition used in the Basilar Artery International Cooperation Study (BASICS) registry [9].

\section{Endovascular Procedure}

The endovascular procedure was performed under general anesthesia, through a transfemoral arterial approach, using an 8-F guide catheter (Neuron Max 6F 088 inches), a 5-F or 6-F intermediate catheter (Sofia plus, ACE 68, Catalyst, and 4MAX), and a microcatheter (3MAX, Velocity, Headway 0.27, Headway 0.21, and AXS Offset) advancing over a microwire (Transend EX Floppy 0.14 and avigo 0.14). Thrombo-aspiration was performed using a pump system for approximately 2 min with the intermediate catheter placed proximally to the occlusion site. Thrombectomy was performed by removing the microwire and putting the stent-retriever (Solitaire stent, Trevo stent, and Revive stent) into the microcatheter. The stent-retriever was opened across the thrombus to engage it and pulled back after 2 min under continuous aspiration applied by the intermediate catheter with the pump system. A Neuroform Atlas stent was placed to treat the underlying stenosis.

\section{Statistical Analysis}

Continuous variables are presented as means and standard deviations (or median and IQR), while categorical variables are presented as absolute and percentage values. The Shapiro-Wilk test was employed to provide evidence that sample data are likely drawn from a normally distributed population. Quantitative variables were compared with the Student's $t$ test and Mann-Whitney $\mathrm{U}$ test for normally and abnormally distributed data, respectively. Categorical variables were compared with the $\chi^{2}$ test or Fisher's exact test as appropriate. We did not perform multivariate analysis because of the small sample size. All tests were 2-sided and $p$ values of 0.05 or less were considered statistically significant. All analyses were performed in the $R$ statistical computing environment, version 3.5.2. 

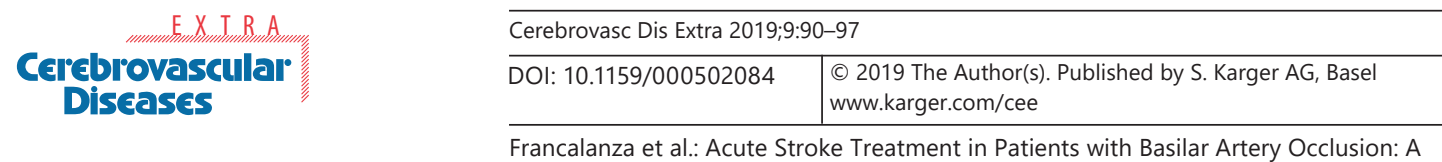

Francalanza et al.: Acute Stroke Treati
Single-Center Observational Study

Table 1. Risk factors, clinical characteristics, and outcome variables

\begin{tabular}{|c|c|c|}
\hline Characteristics/risk factors & $n$ & $\%$ \\
\hline Mean age \pm SD, years & $66 \pm 14$ & \\
\hline Male sex & 16 & 59.3 \\
\hline Hypertension & 18 & 66.7 \\
\hline Hypercholesterolemia & 11 & 40.7 \\
\hline Atrial fibrillation & 6 & 22.2 \\
\hline Diabetes & 7 & 25.9 \\
\hline Current smokers & 9 & 33.3 \\
\hline Mean NIHSS at admission \pm SD & $15 \pm 8$ & \\
\hline \multicolumn{3}{|l|}{ BA segment occlusion } \\
\hline Proximal & 9 & 33.3 \\
\hline Middle & 8 & 29.6 \\
\hline Distal & 10 & 37.0 \\
\hline \multicolumn{3}{|l|}{ Etiology } \\
\hline Large artery disease & 10 & 37.0 \\
\hline Cardioembolic & 3 & 11.1 \\
\hline Unknown & 14 & 51.8 \\
\hline OTT (min.-max.), min & $436(110-1,170)$ & \\
\hline$<6 \mathrm{~h}$ & 12 & 44.4 \\
\hline 6-9 h & 9 & 33.3 \\
\hline $9-12 \mathrm{~h}$ & 3 & 11.1 \\
\hline$>12 \mathrm{~h}$ & 3 & 11.1 \\
\hline$\Delta$ NIHSS $\geq 5$ at discharge & 9 & 33.3 \\
\hline \multicolumn{3}{|l|}{ mRS at 3 months } \\
\hline $0-3$ & 11 & 40.7 \\
\hline $4-5$ & 8 & 29.6 \\
\hline 6 & 8 & 29.6 \\
\hline
\end{tabular}

SD, standard deviation; NIHSS, National Institutes of Health Stroke Scale; BA, basilar artery; OTT, onset to treatment time; mRS, modified Rankin Scale.

\section{Results}

The study population consisted of 27 patients (16 males). The mean age was $66( \pm 14)$ years. Risk factors and clinical characteristics are shown in Table 1.

Five patients came from Messina, 1 from Reggio Calabria, and the others from different parts of Sicily. Of total presentations, $33.3 \%$ of patients presented directly to our emergency department, while $66.7 \%$ were transferred from other hospitals.

We observed a favorable outcome in $40.7 \%$ of cases, and $25.9 \%$ reached mRS $0-2.29 .6 \%$ had a poor clinical outcome (mRS 4-5). Patient survival at 3 months was $70.4 \%(n=19)$, whereas 8 patients died (29.6\%), 5 during hospitalization. The mean NIHSS score at admission was $15( \pm 8)$. The average 0TT was $436 \mathrm{~min}$ (min. 110 to max. 1,170).

Imaging and procedural data are shown in Table 2. In $88.9 \%$, successful recanalization was obtained. The mean pc-ASPECTS was $6( \pm 2)$, in 7 patients pc-ASPECTS was $\geq 7$. According to the PC-CS, $33.3 \%$ had moderate collaterals and $63.0 \%$ had good collateral status prior to receiving treatment. Just 1 patient had bad collaterals. Intracerebral hemorrhage occurred in 2 cases, and neither of them underwent IV-T. In 1 patient, in which additional right brachial artery access was required, brachial artery thrombosis occurred which needed surgical repair. Favorable outcome was significantly associated with age $(p=0.034)$, NIHSS score at admission $(p=0.001)$, pc-ASPECTS $(p=0.005)$, hypercholesterolemia $(p=0.045)$, and female $\operatorname{sex}(p=0.045)$ (Fig. 1). 


\section{Cerebrovascular. \\ Diseases}

Table 2. Imaging and procedural data

\begin{tabular}{l|l}
\hline Cerebrovasc Dis Extra 2019;9:90-97 \\
\hline DOI: 10.1159/000502084 & $\begin{array}{l}\text { @ 2019 The Author(s). Published by S. Karger AG, Basel } \\
\text { www.karger.com/cee }\end{array}$ \\
\hline
\end{tabular}

Francalanza et al.: Acute Stroke Treatment in Patients with Basilar Artery Occlusion: A Single-Center Observational Study

\begin{tabular}{lcc}
\hline Parameters & $n$ & $\%$ \\
\hline Mean pc-ASPECTS \pm SD & $6 \pm 2$ & \\
$\geq 7$ & 7 & 25.9 \\
$<7$ & 20 & 74.1 \\
Mean PC-CS \pm SD & $6 \pm 2$ & \\
Bad collaterals (0-3) & 1 & 3.7 \\
Moderate collaterals (4-5) & 9 & 33.3 \\
Good collaterals (6-10) & 17 & 63.0 \\
Treatment & & \\
Thrombo-aspiration only & 17 & 63.0 \\
Thrombo-aspiration + IA-T & 3 & 11.1 \\
Thrombo-aspiration + IV-T & 2 & 7.4 \\
IV-T only & 1 & 3.7 \\
IV-T + IA-T & 1 & 3.7 \\
Mechanical thrombectomy & 3 & 11.1 \\
Needle-to-recanalization, min & 108 & \\
Recanalization status & & \\
TICI 3 & 19 & 70.4 \\
TICI 2b & 5 & 18.5 \\
TICI 0 & 3 & 11.1 \\
\hline
\end{tabular}

SD, standard deviation; pc-ASPECTS, posterior circulation Alberta Stroke Program Early CT Score; PC-CS, Posterior Circulation Collateral Score; IA-T, intra-arterial thrombolysis; IV-T, intravenous thrombolysis; TICI, Thrombolysis in Cerebral Infarction.

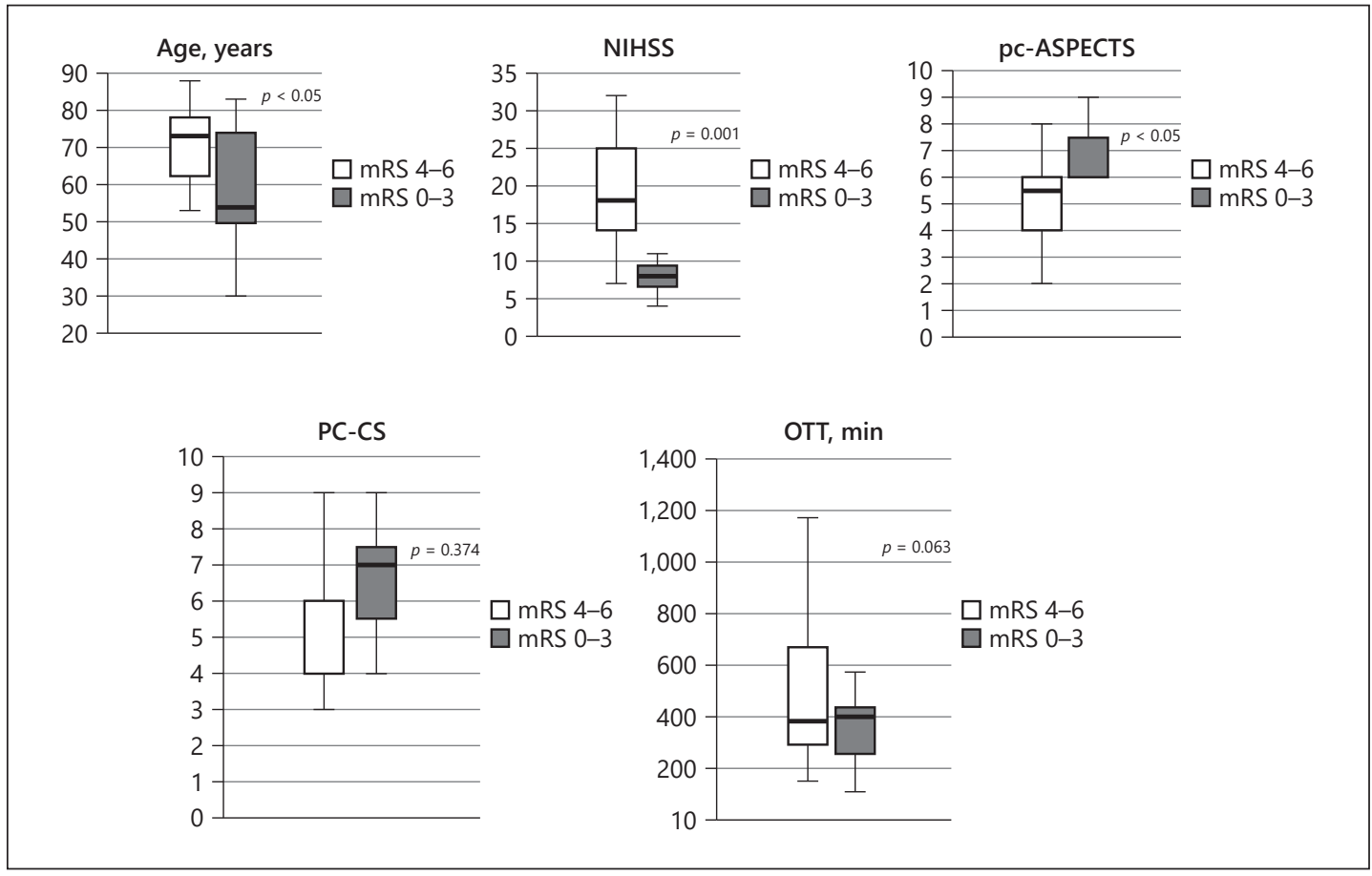

Fig. 1. Univariate analysis of clinical and imaging parameters on functional outcome. NIHSS, National Institutes of Health Stroke Scale; pc-ASPECTS, posterior circulation Alberta Stroke Program Early CT Score; PC-CS, Posterior Circulation Collateral Score; OTT, onset to treatment time. 
The site of occlusion of the BA, whether middle, distal, or proximal, was not associated with functional outcome. Transferred patients had a higher poor outcome rate, but this was not statistically significant $(p=0.26)$. No significant association between functional outcome and collateral status, OTT, and recanalization was found.

\section{Discussion/Conclusion}

In our observational study, mRS 0-3 at 3 months was reached in $40.7 \%$ of cases. Because of the severity of AIS due to BAO, it is reasonable to categorize an mRS score of 3 among favorable outcomes. In the BASIC registry [11], the rate of favorable outcomes was $32 \%$, and in the study by van Houwelingen et al. [20], the rate was $50 \%$. Our results showed a similar rate of death when compared to the literature [13]. $29.6 \%$ of patients did not survive 3 months.

In our study, pc-ASPECTS was associated with functional outcome. Few studies examined DWI data and functional outcome in posterior circulation strokes [15, 21-23]. Tei et al. [15] assessed pc-ASPECTS on DWI according to the method described by Puetz et al. [16] and showed that, in 132 patients with AIS in the posterior circulation, pc-ASPECTS is an independent predictor of favorable outcome (mRS 0-2) [15]. In the study, pc-ASPECTS appeared a powerful marker for predicting functional outcome $(p<0.001)$, suggesting that lesion location and involvement of different territories are more important than lesion volume in influencing functional outcome in posterior circulation patients.

Using the PC-CS, we found no relationship between collaterals and functional outcome. Several studies investigated the prognostic value of collateral status for functional outcome in BAO patients using different scales. Van der Hoeven et al. [17] assessed 149 patients with acute BAO from the BASICS registry and evaluated the association between clinical outcome and collaterals using the PC-CS. They found that $24 \%$ had bad collaterals, $40 \%$ intermediate collaterals, and $36 \%$ a good PC-CS. In contrast to our results, they found that PC-CS independently predicted a poor outcome (mRS 4-6) at 1 month. However, in our study, only $3.7 \%$ had bad collaterals, $33.3 \%$ had moderate collaterals, and $63.0 \%$ had good collaterals. In 24 patients with BAO or BA stenosis $>50 \%$, Alqadri et al. [24] observed that favorable outcome (mRS 0-2) at discharge was associated with good collaterals using a classification system on angiographic images. In accordance with our results, van Houwelingen et al. [20] observed a significant correlation between favorable outcome and both younger age and lower NIHSS, but not for OTT. They found no relationship between outcome and collaterals evaluated on pretreatment cerebral angiography and/or CT angiography imaging, assessing patency of vertebral arteries, posterior communicating artery, and anastomosis between posterior inferior cerebellar artery and superior cerebellar artery. In a large observational multicenter study (ENDOSTROKE analysis), patients with AIS secondary to BAO were examined to evaluate factors associated with outcome and recanalization status. 34\% of patients had a good clinical outcome (mRS 0-2). The mortality rate was 35\%, and successful recanalization was obtained in $79 \%$ of patients. Predictors of clinical outcome were age, hypertension, NIHSS score, use of MRI prior to endovascular treatment, and collateral status evaluated using the American Society of Interventional and Therapeutic Neuroradiology/Society of Interventional Radiology (ASITN/SIR) collateral grading system [13]. In our study, the absence of a relationship between outcome and collateral status should be interpreted with caution. Most of the studies, although using different scores, found collateral status as a prognostic predictor. Collateral flow to the BA may prolong ischemia tolerance and reduce the risk of a poor outcome. 
In our study, the average OTT was $436 \mathrm{~min}$ and OTT did not predict functional outcome. Different studies did not find an association between outcome and time window [13, 20]. However, Vergouwen et al. [25] observed, in 409 patients from the BASICS registry, that OTT $>6 \mathrm{~h}$ is associated with a higher risk of a poor outcome. In our series, large OTT was partly due to the high percentage of transferred patients. $55.6 \%$ of patients were treated beyond $6 \mathrm{~h}$ from symptom onset. Remarkably, we did not find a difference in functional outcome from patients treated within $<6 \mathrm{~h}$ from symptom onset $(p=0.93)$. This finding suggests that an extended therapeutic window is reasonable, taking into account lesion volume and localization in DWI MRI. Delay from symptom onset should not discourage from reperfusion therapies in BAO patients.

Transferred patients had no significant difference in clinical outcome compared to patients presenting directly to our hospital, encouraging the transfer of suspected posterior stroke to the comprehensive stroke center even in extensive OTT.

Major limitations in our study include the observational and retrospective design, together with the small sample size. In conclusion, this study has found that younger age, low NIHSS score at admission, and high pc-ASPECTS, but not OTT, are associated with a favorable functional outcome. This study confirmed that acute stroke treatment improves clinical outcome in BAO patients. Our results encourage a wide recruitment of patients with $\mathrm{BAO}$, in spite of a delayed diagnosis and an extended therapeutic window, considering lesion volume and localization in DWI MRI.

\section{Acknowledgement}

We acknowledge the territorial emergency medical service, which has contributed in an essential way to carrying out the centralization system.

\section{Statement of Ethics}

Subjects (or their parents or guardians) have given their written informed consent. The study protocol has been approved by the research institute's committee on human research.

\section{Disclosure Statement}

The authors have no conflicts of interest to declare.

\section{Funding Sources}

This study did not receive any funding.

\section{Author Contributions}

I.F., P.L.S., and R.F.M. contributed to the study design. I.F. and A.C. performed the data collection. A.A.C., A.P., and S.L.V. performed the imaging analysis. G.T. performed the statistical analysis. C.C., M.C., C.D.A., M.C.F., and F.G. supervised the research. I.F., A.C., P.L.S., and R.F.M. wrote the article. 


\begin{tabular}{l|l}
\hline Cerebrovasc Dis Extra 2019;9:90-97 \\
\hline DOI: 10.1159/000502084 & $\begin{array}{l}\text { (c) 2019 The Author(s). Published by S. Karger AG, Basel } \\
\text { www.karger.com/cee }\end{array}$ \\
\hline
\end{tabular}

Francalanza et al:: Acute Stroke Treatment in Patients with Basilar Artery Occlusion: A Single-Center Observational Study

\section{References}

1 Möhlenbruch M, Stampfl S, Behrens L, Herweh C, Rohde S, Bendszus M, et al. Mechanical thrombectomy with stent retrievers in acute basilar artery occlusion. AJNR Am J Neuroradiol. 2014 May;35(5):959-64.

2 Demel SL, Broderick JP. Basilar Occlusion Syndromes: an Update. Neurohospitalist. 2015 Jul;5(3):142-50.

3 Dornak T, Herzig R, Sanak D, Skoloudik D. Management of acute basilar artery occlusion: should any treatment strategy prevail? Biomed Pap Med Fac Univ Palacky Olomouc Czech Repub. 2014 Dec;158(4):528-34.

4 Goyal N, Tsivgoulis G, Nickele C, Doss VT, Hoit D, Alexandrov AV, et al. Posterior circulation CT angiography collaterals predict outcome of endovascular acute ischemic stroke therapy for basilar artery occlusion. J Neurointerv Surg. 2016 Aug;8(8):783-6.

5 Lindsberg PJ, Pekkola J, Strbian D, Sairanen T, Mattle HP, Schroth G. Time window for recanalization in basilar artery occlusion: speculative synthesis. Neurology. 2015 Nov;85(20):1806-15.

6 Mak CH, Ho JW, Chan KY, Poon WS, Wong GK. Intra-arterial revascularization therapy for basilar artery occlusion-a systematic review and analysis. Neurosurg Rev. 2016 Oct;39(4):575-80.

7 Hacke W, Zeumer H, Ferbert A, Brückmann H, del Zoppo GJ. Intra-arterial thrombolytic therapy improves outcome in patients with acute vertebrobasilar occlusive disease. Stroke. 1988 Oct;19(10):1216-22.

8 Smith WS. Intra-arterial thrombolytic therapy for acute basilar occlusion: pro. Stroke. 2007 Feb;38(2 Suppl): 701-3.

9 van der Hoeven EJ, Schonewille WJ, Vos JA, Algra A, Audebert HJ, Berge E, et al.; BASICS Study Group. The Basilar Artery International Cooperation Study (BASICS): study protocol for a randomised controlled trial. Trials. 2013 Jul;14(1):200.

10 Mattle HP, Arnold M, Lindsberg PJ, Schonewille WJ, Schroth G. Basilar artery occlusion. Lancet Neurol. 2011 Nov;10(11):1002-14.

11 Schonewille WJ, Wijman CA, Michel P, Rueckert CM, Weimar C, Mattle HP, et al.; BASICS study group. Treatment and outcomes of acute basilar artery occlusion in the Basilar Artery International Cooperation Study (BASICS): a prospective registry study. Lancet Neurol. 2009 Aug;8(8):724-30.

12 Nagel S, Kellert L, Möhlenbruch M, Bösel J, Rohde S, Ringleb P. Improved clinical outcome after acute basilar artery occlusion since the introduction of endovascular thrombectomy devices. Cerebrovasc Dis. 2013;36(56):394-400.

13 Singer OC, Berkefeld J, Nolte CH, Bohner G, Haring HP, Trenkler J, et al.; ENDOSTROKE Study Group. Mechanical recanalization in basilar artery occlusion: the ENDOSTROKE study. Ann Neurol. 2015 Mar;77(3):415-24.

14 Sheng K, Tong M. Therapy for acute basilar artery occlusion: a systematic review and meta-analysis. F1000Res. 2019 Feb; 8:165.

15 Tei H, Uchiyama S, Usui T, Ohara K. Posterior circulation ASPECTS on diffusion-weighted MRI can be a powerful marker for predicting functional outcome. J Neurol. 2010 May;257(5):767-73.

16 Puetz V, Sylaja PN, Coutts SB, Hill MD, Dzialowski I, Mueller P, et al. Extent of hypoattenuation on CT angiography source images predicts functional outcome in patients with basilar artery occlusion. Stroke. 2008 Sep; 39(9):2485-90.

17 van der Hoeven EJ, McVerry F, Vos JA, Algra A, Puetz V, Kappelle LJ, et al.; BASICS registry investigators. Collateral flow predicts outcome after basilar artery occlusion: the posterior circulation collateral score. Int J Stroke. 2016 Oct;11(7):768-75.

18 Higashida RT, Furlan AJ, Roberts H, Tomsick T, Connors B, Barr J, et al.; Technology Assessment Committee of the American Society of Interventional and Therapeutic Neuroradiology; Technology Assessment Committee of the Society of Interventional Radiology. Trial design and reporting standards for intra-arterial cerebral thrombolysis for acute ischemic stroke. Stroke. 2003 Aug;34(8):e109-37.

19 Kolominsky-Rabas PL, Weber M, Gefeller O, Neundoerfer B, Heuschmann PU. Epidemiology of ischemic stroke subtypes according to TOAST criteria: incidence, recurrence, and long-term survival in ischemic stroke subtypes: a population-based study. Stroke. 2001 Dec;32(12):2735-40.

20 van Houwelingen RC, Luijckx GJ, Mazuri A, Bokkers RP, Eshghi OS, Uyttenboogaart M. Safety and outcome of intra-arterial treatment for basilar artery occlusion. JAMA Neurol. 2016 Oct;73(10):1225-30.

21 Kim JG, Lee D, Choi JC, Song Y, Lee DH, Suh DC. DWI-pc-ASPECT score in basilar artery occlusion: is 6 points or less always indicative of a bad outcome? Interv Neuroradiol. 2019 Aug;25(4):371-9.

22 Karameshev A, Arnold M, Schroth G, Kappeler L, Stein P, Gralla J, et al. Diffusion-weighted MRI helps predict outcome in basilar artery occlusion patients treated with intra-arterial thrombolysis. Cerebrovasc Dis. 2011; 32(4):393-400.

23 Guillaume M, Lapergue B, Gory B, Labreuche J, Consoli A, Mione G, et al.; Endovascular Treatment in Ischemic Stroke (ETIS) Investigators. Rapid Successful Reperfusion of Basilar Artery Occlusion Strokes With Pretreatment Diffusion-Weighted Imaging Posterior-Circulation ASPECTS [\{LT $\}$ ] Is Associated With Good Outcome. J Am Heart Assoc. 2019 May;8(10):e010962.

24 Alqadri S, Adil MM, Watanabe M, Qureshi AI. Patterns of collateral formation in basilar artery steno-occlusive diseases. J Vasc Interv Neurol. 2013 Dec;6(2):9-13.

25 Vergouwen MD, Algra A, Pfefferkorn T, Weimar C, Rueckert CM, Thijs V, et al.; Basilar Artery International Cooperation Study (BASICS) Study Group. Time is brain(stem) in basilar artery occlusion. Stroke. 2012 Nov; 43(11):3003-6. 